\title{
La evaluación formativa en la educación superior
}

\section{Formative assessment in higher education}

DOI: $10.46932 /$ sfjdv3n1-074

Received in: Jan 30st, 2021

Accepted in: Feb 1th, 2022

\author{
Bazán Linares Magda Veronica \\ Magister \\ Universidad Agraria de la Selva \\ Jr. Sucre 385: Tingo María \\ E-mail: magda.bazan@unas.edu.pe \\ Lauriano Antonio Zavaleta de la Cruz \\ Magister \\ Universidad Agraria de la Selva \\ Calle las almendras. Lote 2. Castillo Grande. \\ E-mail: lauriando.zavaleta@unas.edu.pe \\ Oscar Melanio Dávila Rojas \\ Doctor \\ Universidad Privada César Vallejo \\ E-mail: odavilar25@ucvvirtual.edu.pe

\section{José Ángel Meneses Jiménez} \\ Doctor \\ Universidad Privada César Vallejo \\ Car. Federico Basadre KM. REF: Ucayali Coronel Portillo-Calleria \\ E-mail: jameneses@ucvvirtual.edu.pe

\section{Mabel Cruz Palma Oyola} \\ IES Huando \\ Asociación nuevo san juan Mz A Lt. 42. Puente Piedra \\ E-mail: mailto:agrosostperu@gmail.com
}

\section{RESUMEN}

La evaluación es desde siempre un importante mecanismo de valoración de los aprendizajes en los procesos académicos. Este artículo asumió el objetivo analizar la importancia de la valuación formativa en la educación superior. La investigación siguió el paradigma interpretativo, enfoque cualitativo, documental y explicativo. Para lo cual se realizó una exploración sobre el estado del arte de diferentes artículos científicos sobre la valoración formativa en la educación superior. Una exploración de la importancia de los instrumentos y herramientas que apoyan esta valoración, su predominio en la consecución de las competencias profesionales en los jóvenes universitarios. Se revisaron treinta y cuatro documentos alojados en revistas como Scopus, Wep of Science, Sciencedierct, Scielo, Redalyc, Dialnet y ScienceDirect, manejando discernimientos de elección y excepción. Se percibió que la valoración formativa y sus instrumentos deben formar parte del sistema de evaluación universitaria. Se concluyó, que interviene en el fortalecimiento de la participación colaborativa, el intercambio de información 
promueve su participación crítica de los estudiantes. La rúbrica, el portafolio, la retroalimentación favorecen la autoevaluación, autoformación y el constante estudio. Jóvenes empoderados y más seguros al momento de sus participaciones y esto se refleja en la mejora de las competencias profesionales.

Palabras clave: Evaluación formativa, rúbrica, portafolio, retroalimentación, trabajo colaborativo, competencia profesional.

\begin{abstract}
Evaluation has always been an important mechanism for evaluate the academic learning processes. This article had been has aimed to analyze the importance of formative assessment in higher education. The research followed the interpretive paradigm, qualitative, documentary and explanatory approach. Therefore, it was carried out an exploration of the art state in different scientific articles about formative assessment in higher education. An analysis of the importance about the instruments and tools that support the assessment and their influence on the profession achievement skills in university students. In this research were revised thirty-four documents housed in journals such as Scopus, Wep of Science, ScienceDierct, Scielo, Redalyc, Dialnet and ScienceDirect were reviewed, handling discernments of choice and exception. It was perceived that the formative assessment and its instruments should be part of the university evaluation system. It was concluded that it intervenes in the strengthening of collaborative participation, the exchange of information, promotes its critical participation of students. La rúbrica, el portafolio, el feedback favorecen la autoevaluación, la autoformación y el estudio constante. Jóvenes empoderados y más seguros a la hora de su participación y esto se refleja en la mejora de las competencias profesionales.
\end{abstract}

Keyword: Formative assessment, rubric, portfolio, feedback, collaborative work, professional competence.

\title{
1 INTRODUCCIÓN
}

En el contexto actual donde la globalización ha tocado a todas esferas del quehacer humano se torna cada vez más rigurosa la necesidad de estar acorde con estas exigencias. Esto también ha influenciado en el campo de la educación en donde se necesita contar con profesionales cada vez más preparados y capacitados. Estas pretensiones exigen a los establecimientos superiores a cambiar sus políticas educativas con miras a capacitar a los futuros profesionales en las competencias y habilidades requeridas por la globalización.

En el estudio de Salas, (2019) en el que se analizó la evaluación de procesos en la Universidad. Se pudo notar que esta influye en la adopción y entrega de mejores productos y concluye que hay evidencias que la evaluación formativa logra influenciar en la consecución de resultados óptimos en lo referente a desarrollo académico en estudiantes universitarios. Si bien la evaluación es el procesamiento de información y el juicio que se emite sobre los aprendizajes, entonces evaluar es emitir una valoración basados en observaciones y procesos sistemáticos, estos procesos implican una planificación, ejecución y evaluar el mismo proceso de evaluación (Prieto, 2008). Con la información recogida se pueden tomar acciones oportunas para lograr la mejora constante de los aprendizajes. 
Esta evaluación sitúa al universitario como eje central, es decir como la médula del desarrollo de los aprendizajes. Este modelo se viene aplicando en países como China, países bajos, España, Costa Rica, Cuba, México entre otros, que han intentado poner en marcha este tipo de evaluación, muy necesaria para la mejora del logro de competencias académicas en los estudiantes universitarios. Sin embargo, debemos evaluar ciertos aspectos respecto a este tipo de evaluación, los alcances, si ayuda al desarrollo de los aprendizajes, si cumple o no con la mejora de las competencias académicas (Martínez et al., 2018). Un estudio reciente realizado en China en un estudio que analizó respecto a este modelo formativo aplicándolo en la enseñanza en el curso de Morfología Humana Normal en estudiantes de medicina. Las evidencias revelaron que la evaluación formativa provoca en los estudiantes mayor conocimiento, mejor entendimiento frente a la evaluación tradicional. (Xiaotong et al., 2019).

En algunos países latinoamericanos hay una constante preocupación por valorar los instrumentos de evaluación formativa, donde el estudiante sea el centro del proceso y que se vean favorecidas o fortalecidas sus procesos de aprendizaje. Entonces la apreciación crítica funcionaría en el desarrollo de la instrucción y aprendizaje, permite al docente realizar el acompañamiento constante que potencian del avance de prestezas durante el desarrollo de su educación y en la consecución de sus aprendizajes (Hernández \& Salicetti, 2018).

Las instituciones universitarias peruanas no se han mantenido al margen de estos cambios e innovaciones y la adecuación a estos sistemas de evaluación se viene implementando con el objeto de favorecer los procesos de aprendizaje y se ha configurado y planteado como un reto para la educación actual Chávez et al. (2021). Por tanto, es importante que los docentes se adapten y adopten ciertas estrategias de evaluación y se logra obtener así resultados exitosos en la aplicación evaluación formativa y se ve reflejada en los saberes.

Rodríguez \& Salinas (2020) señalan la evolución de la estimación formativa y cómo se ha modificado en los últimos años, un aspecto a considerar es la implementación de la denominada retroalimentación. Este instrumento permite hacer óptima la innovación, creatividad, un mayor desarrollo de habilidades dentro del proceso de los aprendizajes, empoderamiento para afrontar nuevos retos. Mayor capacidad de respuesta a la hora de resolver situaciones problemáticas planteadas por el contexto social.

Inga et al. (2019) en su trabajo sobre educación, en base a las destrezas que se necesita para una valoración genuina en la universidad señalan que, la apreciación valorativa se debe realizar frecuentemente. En este mismo estudio concluye que la evolución de este modelo de evaluación garantiza la adquisición de competencias y esto propiciará un desempeño óptimo en las funciones que se le encomiende y contribuye a fortalecer las competencias que se buscan en el perfil de los egresados. Otro factor trascendental y que resulta muy apropiado a nivel educativo es la coevaluación como instrumento, 
de tal suerte que el encargado de realizarlo notará los aspectos positivos y las deficiencias que posee (Torres et al., 2021). El proceso de coevaluación permite extraer conclusiones para la mejora de los aprendizajes por lo tanto debe ser realizada siguiendo algunas pautas establecidas pues en base a esto se tomarán medidas y propondrán modificaciones para lograr estas mejoras (Torres et al., 2021).

La apropiación del portafolio en el desarrollo de los aprendizajes es que se debe impulsar el desarrollo del aprendizaje autónomo. Se puede introducir como una herramienta que propicie y a la vez suscite la adquisición de conocimientos en el estudiante quien con la orientación del docente realizarán el plan del portafolio para hacerlo tangible y evidente en la práctica, mostrando de esta manera, avances en el progreso de competencias profesionales que se van adquiriendo en el transcurso del proceso de formación (Salazar \& Arévalo, 2019).

Este trabajo busca analizar la significación de la valoración formativa y sus herramientas para la formación superior con el ánimo de alentar su práctica constante y así mejorar los entornos educativos. Desde hace algunos años, en las universidades se están cambiando los programas de estudio, pasando de los tradicionales a un programa por competencias. Este estudio presentará una recopilación pormenorizada de las experiencias resultantes de la aplicación de este modelo de valoración. Así se contribuye a fomentar las competencias profesionales en jóvenes universitarios.

En este estudio se estimó conveniente examinar la significación de la evaluación formativa en la educación superior. También se plantearon cuatro objetivos: (i) identificar el aporte de retroalimentación a la evaluación formativa. (ii) evaluar la utilidad de la rúbrica en la evaluación formativa. (iii) explicar la importancia del portafolio en el desarrollo de las competencias profesionales. (iv) explicar la importancia del trabajo colaborativo en el desarrollo de las competencias profesionales.

\section{MÉTODO}

En estos tiempos los modelos de enseñanza-aprendizaje han sido objeto de cambios importantes, en los cuales es necesario hacer cambios en los perfiles de los docentes y educandos; el rol del maestro también ha sufrido cambios importantes en estos tiempos. Estos roles han tenido que virar hacia un rol de facilitador de la información, en cambio los estudiantes son ahora más proactivos, no son simples oyentes, ahora participan y son críticos de los procesos.

La exploración cualitativa educacional universitaria busca responder a estos nuevos retos derivados de la globalización, en el cual el estudiante debe generar también sus propios aprendizajes, esta investigación se centra en el análisis descriptivo. Dentro de sus características podemos decir que no trata de probar teorías ya existentes, si no que genera sus propias hipótesis y teorías, apela a la intuición del investigador, los hechos estudiados son cambiantes, el investigador participa relacionándose, 
interactuando con los participantes (Iño, 2018). Según los modelos educativos podemos abordar esta temática desde el aspecto interpretativo. En este proceso el sujeto que realiza la investigación produce, elabora y hace explicaciones fundamentadas en bases teóricas, esto en función de los datos que le proporciona el proceso investigativo (Naupas et al., 2018). El paradigma en al que se sustenta este trabajo de investigación es el interpretativo, pues permite la reflexión, intenta comprender la realidad de hechos observables en la que interactúan las personas, en un contexto determinado. Trabaja con datos cualitativos, los cuales son recolectados (Vasilachis et al., 2006). Se basa en interpretar la conducta humana.

Este trabajo se basó en un diseño documental, se realizó una búsqueda minuciosa de apuntes secundarios, se tomó de documentos de otros indagadores y los datos obtenidos se analizaron e interpretaron. (Arias, 2012). Esta búsqueda nos proporcionó material suficiente para realizar un análisis pormenorizado de un tema sobre el cual se está escribiendo. En esta revisión bibliográfica se seleccionó, se buscó de manera minuciosa las de fuentes útiles para el trabajo, luego se revisó la información, se clasificó, se ordenó, para luego hacer un análisis de la información, según los objetivos y finalmente hacer las conclusiones de la investigación (Guerrero \& Guerrero, 2014).

El alcance de la investigación explicativa, pues para realizar y desarrollar el trabajo de investigación se basó en la revisión de documentos, en primer lugar, se realizó una exploración acuciosa e íntegra de material científico relacionado al tema como menciona (Izcarra, 2014) el cual detallaremos en los siguientes párrafos. Rizo (2015) indica que esta investigación compila información, la sistematiza y la registra. Se debe resaltar que la búsqueda se realizó en fuentes de información primaria.

La metodología utilizada para documentar el material consultado se evaluó, revisó y se organizó detalladamente la información de artículos científicos relacionados con aspectos de evaluación formativa, retroalimentación, portafolio, coevaluación, en artículos científicos. Se trabajó con el software Mendeley, este gestor permitió hacer las referencias bibliográficas y una especie de base de datos con la información recogida (López, 2014). La búsqueda de la información fue realizada artículos científicos de enfoque cualitativo. También se empleó tamices como año de publicación no mayor a 5 años, idioma inglés, la bibliografía consultada se recogieron de bases de datos proporcionados por Scielo, Latindex, EBSCO, SCOPUS, Dialnet, Science Direct. La exploración en la base de datos se efectuó empleando las palabras clave, aprendizaje, evaluación formativa, importancia evaluación, rúbrica, utilidad, estrategia de evaluación y la ayuda de operadores AND, OR y NOT. Estos permitieron optimizar la búsqueda de la información, siendo de mucha utilidad, ahorrando tiempo (Moraga et al., 2014).

Este trabajo permitió empaparse de las condiciones y de cómo se realizan y desarrolla la evaluación formativa universitaria que fueron publicados en revistas de alto impacto, para luego analizar e interpretar la información de los resultados en base a las experiencias en los diferentes estudios realizados, así poder 
llegar a obtener los conocimientos respondan o que brinden una solución al problema observado en la investigación.

\section{RESULTADOS}

\subsection{EVALUACIÓN FORMATIVA EN LA EDUCACIÓN SUPERIOR}

En el estudio en el que se indagaba acerca de la aplicación de la valoración instructiva en los diferentes entornos educativos se encontró que es una herramienta indispensable que unifica el proceso didáctico mediante la comunicación constante entre estudiante-docente. Ésta provoca la evolución integral de los jóvenes, los capacita para ser más seguros, autónomos, ser críticos de sus progresos mediante la autovaloración y coevaluación. Propicia un incremento en los niveles de aprendizaje y conocimientos de los estudiantes (Bizarro et al., 2021). Proporciona información a los docentes sobre avances de sus estudiantes, dificultades que pudieran presentar, aspectos a mejorar, para brindar la ayuda oportuna. También sirve para hacer algunos cambios y reajustes en el plan de estudios, ya que esta se asocia a la mejora de los aprendizajes como refiere (Pérez et al., 2020).

Esta evaluación pretende con el uso de estrategias lograr una mejora de los aprendizajes y que estos sean continuos, un tipo de evaluación personalizada, en la que se miden los aprendizajes de forma individualizada, esto obedece a una programación establecida y planificada (Solano-García et al., 2018). La evaluación por competencias es una práctica que se divisa como innovadora en el ambiente universitario, valora de manera integral las competencias alcanzadas. Según (Amurrio, 2017) la valoración es exhaustiva, donde la evaluación por competencias se transforma en un proceso dinamizador, lógico, activador de la estimación de los resultados conseguidos.

Luna (2019) presenta la evaluación formativa como un modelo en las instituciones universitarias, en los que hubo grupos de discusión, en este trabajo se nuestra la evaluación como importante y necesaria. Desde una mirada armónica reconoce y unifica distintas capacidades de este modelo, también algunas peculiaridades propias del contexto. Esto posibilita aunar esfuerzos para incrementar la eficacia en la instrucción de los jóvenes decurso de su estancia en aulas universitarias.

A partir de la perspectiva de los jóvenes universitarios la evaluación formativa según el estudio de (Souto et al., 2020) señalan que estos sistemas de calificación fortalecen el aprendizaje, este es más diligente, más específico, también aportan que con este sistema hay mayor asimilación en los conocimientos. En los estudiantes de psicología clínica se muestra que este sistema de valoración y sus herramientas tiene mucha significación para reconocer aquellas áreas en la que se requiera un mayor impulso y se deduce que esta evaluación formativa, su monitoreo, la constante supervisión contribuye a mejorar el desarrollo profesional (Belaunzaran, 2019). 
En un estudio de Hortigüela et al. (2017) en los que se analiza la preminencia de los puntos de vista de la valoración y la estrategia metodológica usada en una universidad en España. Los estudiantes señalan que permite implicarse en las actividades, aunque esto signifique realizar una mayor cantidad de trabajos. Les brinda la apertura de colaborar y decidir en clase, optando por este modelo en lugar de la educación tradicional de memorización de contenidos esto fortalece en ellos la capacidad de autonomía. Al realizar una evaluación del proceso los estudiantes no sienten tanta presión (Zabalza \& Lodeiro, 2019).

Schellekens et al. (2021) indaga sobre la creencia de instaurar una evaluación que favorezca supremamente a los estudiantes. Los estudiantes y maestros desarrollan un vínculo de mutua colaboración, comparten roles esta hace que se desenfoque la atención centrada en al maestro hacia la figura del estudiante. El docente toma el rol de orientador y los estudiantes interactúan como socios en lugar de simples oyentes. Se propicia la colaboración, participación, la negociación, discusión generada entre el docente y el estudiante, esto le convierte en socio del proceso evaluativo, adquieren mayor comprensión, aprendizajes más sólidos y positivos.

Ríos \& Herrera (2017) por su parte evaluaron el papel de esta valoración y su relación con los perfiles curriculares en el ámbito laboral. En él se sostiene la importancia de ejecutar e implementar instrumentos para establecer el impacto sobre el aprendizaje por competencias. Los hallazgos fueron que estás competencias logran los saberes: saber hacer, saber estar, saber ser al presentar e informar los resultados de manera constante. Esto los capacita para desenvolverse con eficacia en toma de decisiones, dentro del entorno educativo y laboral.

\subsection{RETROALIMENTACIÓN EN LA EVALUACIÓN FORMATIVA}

Es interesante cómo en estos tiempos se viene investigando sobre las estrategias que se deben utilizar en valoración formativa, que esté orientada a conducir a los estudiantes a la consecución de sus aprendizajes. En tal sentido se plantean y desarrollan algunos modelos los cuales deben contener criterios que logren que los jóvenes se involucren, reflexionen y tengan una actitud crítica de los productos de cómo realizan y entregan sus evidencias. Estos instrumentos deben ser elaborados con mucho cuidado y atendiendo a las necesidades y que cumplan su rol formativo (Molina-Soria et al., 2020).

Para Schildkampa et al. (2020) en su estudio que indaga sobre los requisitos previos para el uso eficaz de la valoración formativa por parte de los docentes, se encontraron que deben poseer conocimientos del contenido a enseñar, cómo enseñar. Se resalta el rol de los docentes en este proceso que va desde la forma como elaboran los instrumentos de evaluación, su uso, la devolución de las tareas encargadas, la retroalimentación oportuna contribuye a reafirmar los aprendizajes. Hay una preocupación en cuanto a la formación y logros destacados y que ésta depende de aplicar bien los instrumentos y que 
realmente se hayan alcanzado las competencias y no sea solamente un evaluar con calificaciones altas y el estudiante tenga una preparación profesional escasa (Salas \& Vicente, 2020).

Ureña \& López (2019) aplican el modelo de evaluación formativa, compartida y de clase donde el estudiante sigue su propio ritmo de trabajo, donde se evaluó además las competencias profesionales. Los hallazgos fueron que los jóvenes obtuvieron calificaciones satisfactorias, cuando la retroalimentación recibida fue hecha de manera oportuna. En el trabajo (Bizarro et al., 2021) respecto a la valoración formativa en los diferentes entornos educativos y en la universidad encontramos que la retroalimentación es fundamental en estos procesos. Ello contribuye a fortalecer la formación integral de los jóvenes, adquiriendo mayor empoderamiento, mejorando su expresión oral y sus niveles de escritura lo que propicia un aprendizaje significativo.

Para alcanzar resultados satisfactorios es necesario la intervención constante y oportuna de los docentes. Que los estudiantes perciban que hay un interés y compromiso en ayudar a la mejora constante, que estos procesos de retroalimentación destaquen los logros obtenidos, identifiquen las dificultades, se brinde aportes y soluciones pertinentes para conseguir superar estas dificultades. Esto genera en el estudiante mayor seguridad, mayor empoderamiento (Álvarez-Iguaín \& Torres-Belma, 2021). En el estudio de (Canabal \& Margalef, 2017) se puede elucidar que los docentes recogieron evidencias que les permite adecuar y ajustar sus contenidos, si fuese necesario redelinear sus estrategias. Entonces la retroalimentación incrementa la motivación y propicia que los aprendizajes continuos siempre y cuando estén bien orientados y propicien el diálogo y la reflexión. (

Los hallazgos fundamentales se resalta el rol del docente al aplicar esta retroalimentación oportunamente, lo que se refleja en la mejora en sus productos. Después de la retroalimentación mostraron mayor motivación para propiciar su autoaprendizaje, se notó una mejora en las evaluaciones. Se observó mayor participación en las sesiones de aprendizaje, incluso sus intervenciones acertadas incrementaron el nivel de discusión. Finalmente, al propiciar mayor reflexión, más motivación, mayor intercambio de ideas hace que la educación sea continua.

\subsection{EVALUAR LA UTILIDAD DE LA RÚBRICA EN LA EVALUACIÓN FORMATIVA}

La rúbrica por otro lado se constituye en otra herramienta versátil en evaluación y permite que los maestros puedan interactuar con sus estudiantes. Fomenta que los estudiantes sean más reflexivos, que tengan una visión más profunda del proceso que se evalúa y da pie a la autoevaluación. Del lado de los maestros ayuda al mejoramiento de los planes y programas académicos en las universidades, como el estudio de realizado y aplicado por los docentes especialistas en la experiencia curricular de tecnología educativa en una facultad de ciencias médicas, llevaría a los estudiantes a ser más eficientes y situarse en 
el centro de los avances científicos y académicos para enfrentar satisfactoriamente las exigencias laborales propias de su entorno profesional (Piedra et al., 2018).

Un estudio que se propuso homologar y la validación del instrumento que permite estimar la forma en que realiza la valoración de los conocimientos, basado en el modelo por competencias. Se incorpora la retroalimentación con el propósito de involucrar a los estudiantes ofreciéndoles las orientaciones necesarias para mejorar sus aprendizajes, sus competencias. Se pudo determinar que aporta a la mejora de la evaluación en los aprendizajes y recomienda su utilización en otros contextos culturales (Hebles et al., 2017). En el estudio de (García-Valcalver et al., 2020) para validar una rúbrica en estudiantes de maestría se desprende que propicia espacio para la reflexión en base a los resultados logrados, estimulan la profundización de sus conocimientos y de mayor calidad. Esta ofrece al docente la posibilidad de una valoración más justa, con mayor objetividad y al estudiante un medio valioso para la mejora constante.

Respecto a la utilidad de la rúbrica en los procesos de valoración de los aprendizajes hace que los alumnos analicen reflexivamente los procesos de evaluación. Esta herramienta brinda la información de cómo se están desarrollando los niveles de desempeño de los estudiantes. Propicia a los maestros la toma oportuna de decisiones que permitan mejorar algunos aspectos de los procedimientos en la enseñanza de aprendizajes.

\subsection{PORTAFOLIO EN EL DESARROLLO DE LAS COMPETENCIAS PROFESIONALES}

Según Salazar \& Arévalo (2019) en las que analiza la inserción del portafolio como herramienta en educación, se analizaron los documentos bibliográficos, según los datos desprendidos de la exploración estableció que el portafolio es una especie de facilitador del progreso en el perfeccionamiento de las técnicas de aprendizaje y enseñanza. Pone en evidencia el de manera práctica los progresos y consecución de competencias profesionales que en su formación académica.

Ciesielkiewicz (2019) Es su estudio intenta valorar la percepción de los estudiantes sobre esta herramienta, de manera digital, se desprende que los estudiantes todos ellos futuros maestros están dispuestos a utilizar esta herramienta en el futuro en su práctica docente. Se puede decir que es una herramienta valiosa y que al elaborarlo se está en constante estudio, esto contribuye con la consecución de las competencias profesionales.

Muñoz (2020) en que se analizó el carácter, razón del portafolio digital, su influjo que despliega en la metodología, tácticas de enseñanza. La influencia en la edificación de los conocimientos, el interés y satisfacción, la complacencia de los estudiantes, así como la manera en que incide en los aprendizajes. Se concluye que es una herramienta que implica el armonizar estrategias ágiles, didácticas y 
organizacionales y que promueve el desarrollo de competencias capaz de hacer frente los retos que plantea este siglo.

Matsumoto-Royo \& Cid-Galán (2021) en su investigación que pretendía identificar las estrategias de enseñanza y procesos de evaluación basados en la práctica pedagógica usadas en la universidad, ubica al portafolio en los procesos de evaluación y explican cómo el uso de estas carpetas personales se refleja en los aprendizajes, brinda oportunidades para la indagación, discusión y reflexión. Generando un impacto positivo durante el transcurso enseñanza aprendizaje.

En los trabajos revisados se pudo encontrar que el portafolio constituye un instrumento importante en el entorno universitario, facilitando la evidencia práctica de los avances de los estudiantes. Facilita el aprendizaje autónomo y se evidencia la puesta en práctica de los aprendizajes logrados. En la evolución de valoración formativa se presenta a manera de herramienta que admite el seguimiento de las actividades academicistas. El portafolio contiene todo el trabajo realizado por los estudiantes y con el aporte de los docentes. Otro hallazgo es que contribuye a desarrollar las competencias profesionales al estar en constante elaboración y revisión de los contenidos.

\subsection{EL TRABAJO COLABORATIVO EN EL ENFOQUE POR COMPETENCIAS}

Martín-Salinas \& Cid-Galán (2018) destacan la experiencia en el aprendizaje colaborativo y la necesidad de realizar una evaluación donde los estudiantes sean como sean los entes dirigentes de la evaluación, pero también son receptores de los contenidos de la evaluación. Este modelo fue aplicado en jóvenes alumnos de enfermería, se notó lo que contribuyó a fomentar e impulsar su participación, desarrollando en ellos la actitud opinante, juiciosa donde se pudo notar el apoyo al trabajo personalizado y colaborativo.

En un estudio de Campo et al. (2020) donde se recogía la impresión sobre estrategias innovadoras en primeros auxilios de los estudiantes de enfermería, el resultado señaló como positiva la metodología, la experiencia de aprender en forma colaborativa, lo que motivó la actitud crítica, seguridad al momento de aplicar sus conocimientos, mayor empoderamiento esto en el área de emergencia de un hospital.

En el estudio del trabajo colaborativo entre pares de los estudiantes de primer año de enfermería, los resultados indican que los estudiantes que realizaron un trabajo en equipo desarrollaron competencias, como la organización y cuidados de enfermería, cooperación, asistencia técnica, documentación en los registros de los pacientes. Se observó que se apoyaban mutuamente a entender y encontrar información en diferentes fuentes, prepara al estudiante para el autoaprendizaje, propicia la discusión y mayor diálogo lo que contribuye a mejorar la calidad de sus competencias profesionales (Pålsson et al., 2021). 
En el trabajo de observar las competencias de futuros profesionales de educación física se determinó que el trabajo colaborativo aporta una gama de aptitudes en relación con las exigencias y obligaciones del docente. También revela que la interrelación con la comunidad favorece las condiciones del diálogo entre pares y que se pueden establecer trabajo de redes colaborativas, lo que propiciará el intercambiar experiencias, manejo de más información, que influenciará directamente en sus competencias profesionales (Henrique et al., 2018).

En relación con el trabajo colaborativo se ha propiciado que los estudiantes sean más participativos, desarrollen su capacidad crítica, se aportan mutuamente y se apoyaban en la búsqueda de información relevante que les ayude a entender y comprender una temática determinada. Esto propició que se involucren en los procesos de evaluación animándonos perseverar en este sistema de trabajo. El hallazgo es muy importante es que se sentían más seguros y con menos estrés para hacer frente incluso evaluaciones. Al realizar el trabajo colaborativo ven desarrollada su capacidad de liderazgo y tener mayor seguridad al momento de aplicar sus conocimientos en su práctica profesional.

\section{DISCUSIÓN}

Al concluir la revisión sistemática de la información asumió el objetivo general: analizar la importancia de la evaluación formativa en la educación superior, se pudo verificar que es una herramienta indispensable, provocando el autoaprendizaje, evaluación, fortalece la capacidad de involucramiento de los estudiantes en las actividades relacionadas con sus propios aprendizajes. En los estudios revisados se ha podido notar que varios autores con inciden en que contribuye a desarrollar niveles más altos de conocimientos, aporta mayor empoderamiento, gestión y fortifica los aprendizajes obteniendo mejores desempeños y mejoran las competencias profesionales, (Bizarro et al., 2021; Pérez et al.,2020; Luna 2019; Souto et al.,2020; Schellekens et al., 2021; Salsas,2019; Chávez et al. 2021). Valora de manera integral las actividades académicas, permite reflexionar sobre los resultados obtenidos, hacer aportes y reajustes pertinentes (Amurrio, 2017; Souto et al.,2020; Belaunzaran, 2019) lo que es coherente con lo expresado por (Prieto,2008). También se ha hecho evidente que el uso de las estragadas debe ser planificado y se recomienda que estas herramientas se deban proporcionar oportunamente a los estudiantes (Belaunzaran, 2019; Solano-García et al., 2018). Algunos autores resaltan la colaboración que se establece con el docente y con los mismos estudiantes (Schellekens et al., 2021; Luna, 2019; de Hortigüela et al., 2017). Sin embargo, en uno de los hallazgos señala que en este tipo de evaluación incrementa la cantidad de trabajos a realizar, pero que eso no genera malestar en los estudiantes (Hortigüela et al.2017). Los sistemas de evaluación formativa evidencian información respecto a los conocimientos y las destrezas que los 
estudiantes alcancen a obtener y su incremento en los niveles de conocimientos. Estos ayudan y fortalecen los saberes capacitándoles para empeñarse mejor en el ámbito educativo y laboral (Ríos \& Herrera, 2017).

La información que se revisó identifica las estrategias de retroalimentación utilizadas en la evaluación formativa, esta contribuye, que desarrollaron una mayor actividad crítica, propicia en ellos una formación continua. Una retroalimentación realizada oportunamente tiende a motivar en el autoaprendizaje y esto se evidencia en las discusiones generadas durante las sesiones de aprendizaje. Los documentos consultados señalan en sus hallazgos ésta debe conducir a los estudiantes a desarrollar una postura capaz de analizar y criticar de sus propios procesos (Molina - Soria et al., 2020; Canabal \& Margalef, 2017). También se pudo notar que después de los procesos de retroalimentación los trabajos y productos desarrollados por los jóvenes habían incrementado significativamente en su calidad (Molina Soria et al., 2020; Ureña \& López, 2019). Un autor señala que existe una preocupación de que los estudiantes realmente no hayan desarrollado competencias profesionales y que sin embargo obtengan calificaciones elevadas (Salas \& Vicente, 2020). Lo cual es contradictorio con los planteados por (Hernández \& Salicetti, 2018; Schildkampa et al., 2020; Molina-Soria et al., 2020) en el cual señala que estos instrumentos son cuidadosamente elaborados y se revisan constantemente contribuyen con su rol formativo. Los docentes deben ser conocedores de la temática a enseñar, conocer cómo brindar ese conocimiento, cómo analizar e interpretar la información recogida para así cumplir con la finalidad de fortalecer los conocimientos (Schildkampa et al., 2020; Bizarro et al., 2021). El propiciar el uso de la rúbrica tiende a desarrollar un importante ambiente de aprendizaje y se propicia un mayor grado de confianza entre el estudiante y docente.

En cuanto la utilidad de la rúbrica en la evaluación formativa es una herramienta primordial brinda información respecto a los niveles logrados de conocimiento respecto a una temática específica. Los hallazgos indican que es muy versátil, posibilita el reconocer los aspectos destacados, los avances y los que aún faltan alcanzar o están proceso de los evaluados, crea una actitud de autoaprendizaje y de lograr mayor profundidad en la información que manejan en torno a sus conocimientos (Piedra et al., 2018; Hebles et al., 2017; García-Valcalver et al., 2020; Bizarro, 2021), por otro lado aporta a los maestros información valiosa para tomar acciones oportunas y pertinentes para mejorar las actividades académicas en las que se requiera profundizar más o en aquellas áreas donde los estudiantes tuvieron un bajo desempeño (Hebles et al., 2017), para conseguir una mejora en las competencias profesionales.

Al concluir el trabajo y explicar la importancia del portafolio en el desarrollo de las competencias profesionales se corroboró: al momento de su elaboración los jóvenes ponen en práctica los conocimientos y proporcionan información de los avances y logros conseguidos. Al desarrollar el portafolio los estudiantes logran una constante revisión de los conocimientos y esa revisión contribuye a 
mejorar la capacidad de respuesta en la aplicación de sus competencias profesionales. En los estudios que se han realizado al respecto de se pudo encontrar que es una herramienta que posibilita y capacita a los estudiantes a realizar un estudio autónomo, propicia y predispone el avance profesional, la consecución de competencias profesionales y el constante estudio (Salazar \& Arévalo, 2019; Ciesielkiewicz, 2019; Muñoz, 2020, Matsumoto-Royo \& Cid-Galán, 2021).

Con relación a la revisión en la que se explica la pertinencia del trabajo colaborativo en el perfeccionamiento de las competencias profesionales se pudo encontrar que motivó la participación constante, aportó en ellos una actitud más crítica, se apoyaban mutuamente en la búsqueda de información necesaria para potenciar sus conocimientos. Así mismo se pudo evidenciar estudiantes más seguros y empoderados, con capacidad de liderazgo lo que se reflejó al momento de aplicar sus conocimientos en su práctica profesional. Los autores consultados coinciden que el trabajo en equipo contribuye a incrementar la participación en el diálogo y discusión, desarrolla su apreciación crítica (Martín-Salinas \& Cid-Galán, 2018; Campo et al.,2020; Martín-Salinas \& Cid-Galán, 2018; Pålsson et al., 2021; Henrique et al., 2018). Sin embargo, en sólo un estudio se pudo encontrar que los jóvenes se ven impulsados a asistir a las sesiones de aprendizaje voluntariamente (Martín-Salinas \& Cid-Galán, 2018). Esto concuerda con lo expresado por (Torres et al.,2021; Rodríguez \& Salinas, 2020) quienes señalan que estos aspectos favorecen las competencias profesionales de los estudiantes.

El implementar la evaluación formativa en este nivel educativo se debe tener en cuenta todos los hallazgos que se ha evidenciado estaban cimentados en la planificación y elaboración cuidadosa y meticulosa de los instrumentos, los cuales deben albergar todos los aspectos que se necesiten para la obtención de resultado fiables. Esto se ve favorecido cuando los docentes utilizan estrategias metodológicas de la evolución formativa y confeccionan su instrumento siguiendo sus lineamientos (Molina - Soria et al., 2020).

Este estudio se limitó a recopilar la información basada en los estudios realizados en los cuales se usó la evaluación formativa en el ámbito universitario en algunos casos futuros docentes, en otros alumnos del área de la salud los cuales por sus características están predispuestos a colaborar con este tipo evaluación y la limitación que se encuentra es que si es posible que se generalice en otros contextos educativos.

La relevancia de la valoración formativa y sus herramientas educacional universitaria crea espacios para seguir investigando, indagar sobre las distintas herramientas utilizadas en la evaluación formativa rúbrica, portafolio, retroalimentación, trabajo colaborativo, en estudiantes universidades extranjeras y del país. Así tener una visión más clara de cómo estos influyen y contribuyen en la mejora de las competencias profesionales. 


\section{CONCLUSIONES}

Las tácticas de la valoración formativa representan el inicio para consolidar y fortificar los aprendizajes, de esta forma se percibió que la valoración formativa fortalece las capacidades y destrezas, aptitudes, pericias de los jóvenes. Las cuales se dan de manera continua en el desarrollo de las sesiones de los aprendizajes. Todo esto haciendo uso de todas las herramientas propias de este modelo de valoración como el trabajo colaborativo, retroalimentación, uso del portafolio, rúbrica que logran fortalecer las competencias profesionales de los jóvenes universitarios.

La valoración formativa se apoya en la retroalimentación y se encontró que constituye una herramienta indispensable en estos espacios. Si este proceso está bien realizado por el docente, después de haber analizado e interpretado los resultados de la rúbrica. El docente hace los aportes pertinentes para potenciar aquellos conocimientos que aún no se han logrado e incluso modificar las estrategias de enseñanza. En los estudiantes genera mayor actitud crítica, autoaprendizaje, despista el interés por mejorar, esto se evidencia en la entrega de sus productos al momento de hacer sus entregas. Los trabajos son elaborados con más cuidado, siguiendo las sugerencias realizadas por los docentes en la retroalimentación. Si la retroalimentación es bien realizada y el docente es un conceder de la temática contribuye con el fortalecimiento de los conocimientos.

Rubrica es un instrumento que apoya la valoración formativa, mantiene a los docentes y jóvenes informados de su proceso. Una vez recogida la información y realizado el análisis el docente puede tomar acciones oportunas para ampliar y mejorar los aspectos en los que los estudiantes tiene mayor dificultad. Insta a los jóvenes tomar conciencia de las falencias, aspectos positivos mediante la autoevaluación. Así ellos mismos van tomando conciencia de sus logros, avances y los que aún están en proceso y tomar acciones para mejorar. Propicia el interés por generar su autoaprendizaje, incrementar y profundizar en sus conocimientos.

Finalmente, el portafolio constituye un elemento que también es importante dentro de la valoración académica, pues recoge las evidencias de las tareas ejecutados por los jóvenes. Se encontró que en su construcción del material los jóvenes aplican todos sus conocimientos y habilidades adquiridas, se mantienen en constante revisión de información. Todo esto propicia en que sus respuestas sean más acertadas y reflexivas al momento de sus participaciones, logrando potenciar sus competencias profesionales. Este trabajo de investigación contribuye con el conocimiento aportando información relevante sobre la importancia de la valoración formativa y sus instrumentos. Fortalecerá las competencias profesionales de los jóvenes y servirá de cimiento para futuras indagaciones. 
Una de las herramientas estudiadas es el trabajo colaborativo y se concluye que ésta promueve la interacción, la unión en el aula; así mismo los jóvenes intercambian y comparten material de clase, informaciones. Se crea entre ellos un clima de mutua colaboración, armonía, ecuanimidad, se propicia espacios para la discusión con la participación activa de los mismos. Los integrantes del grupo logran responsabilizarse y cumplir con las tareas encomendadas de manera satisfactoria y eficiente. Ésta también apoyada por la coevaluación donde recogen los aportes y sugerencias de sus compañeros logran fortalecer las competencias profesionales. Los jóvenes han logrado sentirse más empoderados, seguros al momento de expresar sus ideas, al momento de asumir resoluciones y hacer usos de los conocimientos en su práctica profesional.

\section{RECOMENDACIONES}

En este trabajo se hizo un análisis documental, en la que se recogieron estudios respecto a la valoración que se hizo a los estudiantes, su percepción sobre la evaluación formativa y sus herramientas. La mayor parte de los estudios fueron en jóvenes de ciencias de la salud y educación, faltan por realizar estudios en otras áreas del conocimiento como ingeniería, ciencias sociales en general. Por lo tanto, se recomienda el realizar trabajos en estas áreas del conocimiento en las universidades para determinar, evidenciar su importancia y la medida en la que contribuye a mejorar las competencias profesionales.

En cuanto a la retroalimentación, el portafolio y la rúbrica si bien son instrumentos que aplicados de manera oportuna aportan mucho a la formación de los estudiantes. Sin embargo, sería recomendable que se hagan más estudios en otras áreas del saber para hacer las observaciones necesarias de su influencia en la consecución de las competencias profesionales. Se debe profundizar en la elaboración de la rúbrica, manera de su aplicación y versatilidad. Finalmente se debería incluir en estos estudios a un mayor número de docentes de diferentes especialidades para así tener una visión más amplia de todo este proceso.

En cuanto al trabajo colaborativo hay muchos aspectos que indagar como por ejemplo la manera como se conforman los grupos de trabajo, el número de integrantes, si estos son sugeridos por los docentes o si son por libre elección. Por otra parte, sería bueno hacer un seguimiento y monitoreo de la forma como trabajan los grupos para así propiciar que todos los integrantes logren trabajar cohesionadamente y todos aporten al trabajo.

A modo de recomendación sería conveniente capacitar a los docentes en estas estrategias, como elaborar una rúbrica, qué información nos brinda, como hacer una retroalimentación, afín institucionalizar su aplicación en el ámbito universitario. 


\section{REFERENCIAS}

Amurrio, F. (2017). Evaluación de los aprendizajes por competencias evaluation of competency learning. Revista scientia, 6(1). http://www.revistasbolivianas.org.bo/pdf/risc/v6n1/v6n1_a07.pdf

Álvarez-Iguaín, C., \& Torres-Belma, A. (2021). Percepción de egresados de medicina de una universidad de Chile sobre el cumplimiento de objetivos curriculares del plan de estud. Revista de la fundación educación eédica, 24(2). doi:https://dx.doi.org/10.33588/fem.242.1115

Belaunzaran, J. (2019). EuroPsy: Un modelo basado en competencias. ¿Es aplicable a la formación sanitaria especializada en psicología clínica? Educación médica, 20(2), 154-162.

https://doi.org/https://doi.org/10.1016/j.edumed.2018.05.017

Bizarro, W., Paucar, P., \& Chambi-Mescco, E. (2021). Evaluación formativa: una revisión sistemática de estudios en aula. Revista de Investigación en ciencias de la educación, 5(19), 872 - 891.

https://doi.org/Https://doi.org/10.33996/revistahorizontes.v5i19.244

Campo, W., Rodríguez, B., Formaggi, C., Fernanda de Lima, M., Garanhani, M., \& Féliz de Oliveira, M. (2020). Educational intervention on acute management of burns based on innovative pedagogical methods: Nurses' perceptions. Texto \& Contexto Enfermagem, 29.

https://doi.org/https://doi.org/10.1590/1980-265X-TCE-2019-0222

Canabal, C., \& Margalef, L. (2017). La retroalimentación: la clave para una evaluación orientada al aprendizaje. Revista de curriculum y formación del profesorado, 21(2), 149-170.

https://recyt.fecyt.es/index.php/profesorado/issue/view/3088

Chávez, L., Peña, C., Gomez, S., \& Huayta-Franco, Y. (2021). Evaluación formativa: Un reto en la educación actual. Cuadernos de desarrollo aplicados a las TIC, 10(3). https://doi.org/org/10.17993/3ctic.2021.103.41-63

Ciesielkiewicz, M. (2019). The use of e-portfolios in higher education: From the students' perspective. Villanueva-complutense University of Madrid, 29(3), 649-667. https://doi.org/https://search.informit.org/doi/abs/10.3316/ielapa.641203511753765

Hebles, M., Alonso-Dos-Santos, M., Alvarez-de-Eulate, C. Y., \& Villardon-Gallego, L. (2017). Diseño y validación de la escala evaluación de los aprendizajes (EEA). Revista de currículum y formación de profesorado, 21(2), 107-126. https://www.redalyc.org/pdf/567/56752038007.pdf

Henrique, J., da Silva, J., Januário, C., \& deSouza, S. (2018). Autopercepção de competências profissionais de professores de educação física iniciantes e experientes. Ciências do esport, 40(4), 388396.

https://doi.org/https://doi.org/10.1016/j.rbce.2018.03.020

Hernández, J., \& Salicetti, A. (2018). La evaluación formativa en el proceso enseñanza-aprendizaje en estudiantes de actividad deportiva de la universidad de Costa Rica. Estudios Pedagógicos, XLIV(2).

https://www.redalyc.org/articulo.oa?id=173565234017 
Hortigüela, D., Ausín, V., Delgado, V., \& Abella, V. (2017). Análisis de la importancia de los criterios de evaluación y el reconocimiento académico docente universitario como indicadores de la calidad educativa en España. Revista de la educación superior, 46(181), 75-87. https://doi.org/https://doi.org/10.1016/j.resu.2016.10.002

Inga, M., \& Sánchez, T. (2019). La formación por competencias requiere una evaluación auténtica en la universidad. Revista dilemas contemporáneos: Educación, política y valores, VII(10).

https://doi.org/https://doi.org/10.46377/dilemas.v30i1.1274

Iño, W. (2018). Investigación educativa desde un enfoque cualitativo: La historia oral como método. Voces de la educación, 3(6), 93-103. Obtenido de https://www.revista.vocesdelaeducacion.com.mx/index.php/voces/article/view/123

Izcarra, S. (2014). Manual de investigación cualitativa. Fontamara.

López, M. (2014). Análisis comparativo de los gestores bibliográficos sociales Zotero, Docear y Mendeley: características y prestaciones. Cuadernos de gestión de información., 51-66. https://portal.issn.org/resource/ISSN/2253-8429

Luna, E. (2019). Evaluación formativa del modelo educativo en instituciones de educación superior en México. Revista mexicana de investigación educativa, 24(83), 997-1026. http://www.scielo.org.mx/pdf/rmie/v24n83/1405-6666-rmie-24-83-997.pdf

Martínez, A., Cruz, A., Bamond, V., Fernández, I., \& Strotmann, B. (2018). La observación formativa como instrumento de desarrollo en profesores universitarios en la universidad europea de Madrid. Revista complutense de educación, 29(4), 1365-1380. https://doi.org/https://doi.org/10.5209/RCED.55431

Martín-Salinas, C., \& Cid-Galán, L. (2018). Experiencia de aprendizaje cooperativo en una asignatura optativa del grado en enfermería. Educación médica, 19(5), 288-293. https://doi.org/https://doi.org/10.1016/j.edumed.2017.10.035

Matsumoto-Royo, K., \& Cid-Galán, L. (2021). Core practices in practice-based teacher education: A systematic literature review of its teaching and assessment process. Studies in educational evaluation, 70.

https://doi.org/https://doi.org/10.1016/j.stueduc.2021.101047

Molina-Soria, M., Pascual-Arias, C., \& López-Pastor, V. M. (2020). El rendimiento académico y la evaluación formativa y compartida en formación del profesorado. Alteridad revista de educación, 15(2), 204-215.

https://doi.org/https://doi.org/10.17163/alt.v15n2.2020.05

Monje, C. (2011). Metodología de la investigación cuantitativa y cualitativa guía didáctica. Universidad Surcolombiana.

https://www.uv.mx/rmipe/files/2017/02/Guia-didactica-metodologia-de-la-investigacion.pdf

Moraga, J., Manterola, C., Cartes, R., \& Urrutia, S. (2014). ¿Dónde y cómo buscar evidencia científica en $\begin{array}{lllll}\text { medicina? Revista chilena } & \text { cirugía, } & \text { 66(5), } & \text { 502-507. }\end{array}$ https://doi.org/https://scielo.conicyt.cl/pdf/rchcir/v66n5/art18.pdf 
Muñoz, L. (2020). El portafolio digital educativo sus virtualidades y limitaciones para la enseñanza, el aprendizaje y la evaluación durante la formación Inicial docente [Tesis doctoral. Universidad de Málaga, España]. Repositorio institucional. https://doi.org/https://hdl.handle.net/10630/21917

Ñaupas, H., Mejía, E., Novoa, E., \& Villagómez, A. (2014). Metodología de la investigación cuantitativa - cualitativa y redacción de la tesis. Ediciones de la U.

Pålsson, Y., Mårtensson, G., Swenne, C., Mogensen, E., \& Engstrom, M. (2021). First-year nursing students' collaboration using peer learning during clinical practice education: An observational study. Nurse education in practice, 50. https://doi.org/https://doi.org/10.1016/j.nepr.2020.102946

Pérez, Á., Hortigüela, D., \& Fernandez, J. (2020). Evaluación formativa y modelos pedagógicos: estilo actitudinal, aprendizaje cooperativo, modelo comprensivo y educación deportiva. Revista Española de educación física y deportes, $\mathrm{Vl}(6)$, 44-66. https://www.reefd.es/index.php/reefd/article/\%20view/881

Piedra, I., Eraña, I., Hambleton, A., López, M., \& Segura-Azuara, N. (2018). Delineando criterios para la evaluación de tecnología educativa. Educación médica, 20(2), 108-113. https://doi.org/https://doi.org/10.1016/j.edumed.2018.04.020

Pimienta, J. (2008). Evaluación de los aprendizajes un enfoque basado en competencias. Pearson.

Ríos, D., \& Herrera, D. (2017). Los desafíos de la evaluación por competencias en el ámbito educativo. Educação e pesquisa, 43(3), 1073-1086. https://doi.org/https://doi.org/10.1590/S16784634201706164230

Rodríguez, H., \& Salinas, M. (2020). La evaluación para el aprendizaje en la educación superior: retos de la alfabetización del profesorado. Revista Iberoamericana de evaluación educativa, 13(1), 11-137.

https://doi.org/https://doi.org/10.15366/riee2020.13.1.005

Salas, F., \& Vicente, A. (26 de Julio de 2020). Organización de la evaluación formativa y sumativa: una experiencia. VI Congreso de innovación educativa y docencia en red. Valencia: Conference paper.

https://doi.org/doi:10.4995/INRED2020.2020.11964

Salas, H. (2019). La evaluación formativa orientada al aprendizaje en educación superior a nivel internacional. Yachay, 8(1).

https://doi.org/doi.org/10.36881/yachay.v8i1.131

Salazar, S., \& Arévalo, M. (2019). Implementación del portafolio como herramienta didáctica en educación superior. Revista complutense de educación, 30(4). https://doi.org/https://doi.org/10.5209/rced.59868

Schellekens, L., Bok, H., de Jong, L., van der Schaaf, M., Kremer , W., \& van der Vleuten , C. (2021). A scoping review on the notions of Assessment as Learning (AaL), Assessment for Learning (AfL), and Assessment of Learning (AoL). Studies in educational evaluation, 71. https://doi.org/https://doi.org/10.1016/j.stueduc.2021.101094

Schildkampa, K., Van der Kleijb, F., Heitinka, M., Kippersa, W., \& Veldkampa, B. (2020). Formative assessment: a systematic review of critical teacher prerequisites for classroom practice. International Journal of educational research, 103(101602). https://doi.org/https://doi.org/10.1016/j.ijer.2020.101602 
Solano-García, C., Argota-Pérez, G., Chauca-de-Quispe, C., Pari-Olarte, J., \& Aliaga-Guillen, E. (2018). Conjugación de la práctica para la evaluación formativa del aprendizaje. Biotempo, 15(2). https://doi.org/https://doi.org/10.31381/biotempo.v15i2.2058

Souto, R., Jiménez, F., \& Navarro, V. (2020). La percepción de los estudiantes sobre los sistemas de evaluación formativa aplicados en la educación superior. Revista Iberoamericana de evaluación educativa, 13 (1), 11-39. https://doi.org/https://doi.org/10.15366/riee2020.13.1.001

Torres, J., Chávez, H., \& Cadenillas, V. (2021). Evaluación formativa: una mirada desde sus diversas estrategias en educación básica regular. Revista Innova Educación, 3(2). https://doi.org/ https://doi.org/10.35622/j.rie.2021.02.007

Ureña, N., \& López, P. A. (2019). Evaluación formativa y clase invertida para la adquisición de competencias en el máster de profesorado de educación secundaria. Revista infancia, educación y aprendizaje, 5(2), 470-479. https://doi.org/https://doi.org/10.22370/ieya.2019.5.2.1718

Vasilachis, I., Ameigeiras, A., Chemobilsky, L., Giménez, V., Mallimaci, F., Mendizábal, N., .. . Soneira, A. (2006). Estrategias de la investigación cualitativa. Digesa.

Xiaotong, M., Wujin, C., Libin, L., Tian, L., Wen, Q., \& Shengbin, B. (2019). Analysis of the teaching Effect of the normal human morphology with mixed tteaching mode and formative evaluation in China. International Journal of morphology, 37(3), 1085-1088. https://doi.org/http://dx.doi.org/10.4067/S071795022019000301085.

Zabalza, M., \& Lodeiro, L. (2019). El desafío de evaluar por competencias en la universidad. Reflexiones y experiencias prácticas. Revista iberoamericana de evaluación educativa, 12(2), 29-47. https://doi.org/https://doi.org/10.15366/riee2019.12.2.002 